\title{
ISOTOPISMS OF JORDAN ALGEBRAS ${ }^{1}$
}

\author{
HOLGER P. PETERSSON
}

R. H. Oehmke and R. Sandler have shown in [4] that the middle nucleus of a finite-dimensional semisimple Jordan algebra coincides with its center providing the base field has a characteristic different from 2. By the middle nucleus of a commutative algebra $A$ we mean the set of those elements $x$ in $A$, for which the associator $(y, x, z)$ $=(y x) z-y(x z)$ vanishes identically in $y, z \in A$. Moreover, using this theorem and relying on methods from projective geometry, the authors proved that any two isotopic Jordan division algebras of finite dimension are isomorphic if the characteristic of the base field is also different from 3 . The underlying concept of isotopy in this statement is due to A. A. Albert [1]: Two algebras $A, B$ over the same field are called isotopic if there are bijective linear mappings $\rho, \sigma, \tau$ from $A$ onto $B$ such that $\rho(x) \sigma(y)=\tau(x y)$ for all $x, y \in A$. In this note we intend to establish the following generalizations of the two theorems mentioned above.

ThEOREM 1. Let $A$ be a finite-dimensional semisimple Jordan algebra over a field of characteristic not two. Then each element $x$ in $A$ that satisfies the equation $x^{2} y=x(x y)$ identically in $y \in A$ belongs to the center of $A$.

THEOREM 2. Two isotopic Jordan algebras of finite dimension over a field of characteristic not two, at least one of which is semisimple, are isomorphic.

At the end of the paper we present a short application of these results to a certain generalization of automorphisms.

1. In the sequel, $K$ denotes a (commutative) field of characteristic not 2. If $A$ is an algebra over $K$, it is always tacitly assumed that it has finite dimension. The left multiplication of an element $x$ in $A$ will be denoted by $L(x)$; following [2], we call $P(x)=2 L^{2}(x)-L\left(x^{2}\right)$ the quadratic representation of $x$ in $A$. $A$ is said to be a Jordan algebra providing $A$ is commutative and satisfies the identity $x\left(x^{2} y\right)=x^{2}(x y)$ for all $x, y \in A$. In this case, the (unique) maximal nilideal of $A$ is called the radical of $A$ and is denoted by $\operatorname{Rad} A$. The equation

$$
\operatorname{Rad} A=\{u \in A: u v \text { nilpotent for all } v \in A\}
$$

Received by the editors August 4, 1967 and, in revised form, December 17, 1967.

${ }^{1} \mathrm{I}$ am indebted to the referee for his valuable suggestions and comments. 
is well known [2]. The center of $A$ will be denoted by $Z(A)$. If $c$ is an idempotent of $A\left(c^{2}=c \neq 0\right)$, then $A$ is the direct sum of the subspaces

$$
A_{\lambda}(c)=\{u \in A: c u=\lambda u\}
$$

for $\lambda=0, \frac{1}{2}, 1$. We observe the multiplication rules that $A_{0}(c), A_{1}(c)$ are orthogonal subalgebras of $A$ and $A_{1 / 2}(c) A_{1 / 2}(c) \subset A_{0}(c)+A_{1}(c)$, $A_{\lambda}(c) A_{1 / 2}(c) \subset A_{1 / 2}(c)$ for $\lambda=0,1$.

Proof of Theorem 1. Let $A$ be a semisimple Jordan algebra over $K$. For the moment, we shall call an element $x$ in $A$ alternative if it satisfies

$$
x^{2} y=x(x y)
$$

for all $y \in A$. Let this be the case; in order to prove $x \in Z(A)$ we may assume that $A$ is central simple over $K$. Moreover, the linearity of (1) in $y$ guarantees its validity in every base field extension of $A$. Hence we may assume $K$ being algebraically closed. Then we show

$$
L\left(x^{n}\right)=L^{n}(x)
$$

with induction for all integers $n \geqq 0$. Indeed, the assertion is immediate for $n \leqq 2$, and the induction step is accomplished by the formula $L\left(x^{n+1}\right)=2 L\left(x^{n}\right) \circ L(x)-P(x) \circ L\left(x^{n-1}\right)(n \geqq 2)$ which holds in every Jordan algebra [2, p. 140]. (2) implies $f(L(x))=L(f(x))$ for each polynomial $f$ in one indeterminate $T$ over $K$, from which one easily derives $L\left(f(x)^{2}\right)=L^{2}(f(x))$ for all $f \in K[T]$. Hence every element of the subalgebra $K[x]$ of $A$, generated by the identity element $e$ and $x$, is alternative. Now let $c$ be an idempotent in $K[x]$. Since $L^{2}(c)=L(c)$, we have $A_{1 / 2}(c)=0$, and the decomposition $A=A_{1}(c) \oplus A_{0}(c)$ together with the simplicity of $A$ implies $A_{0}(c)=0$ or $c=e: e$ is the only idempotent of $K[x]$. Finally, let $u$ be an element in $K[x]$ such that $u^{2}=0$. In the linearized Jordan identity

$$
z\left(x^{2} y\right)+2 x((x z) y)=2(x z)(x y)+x^{2}(z y)
$$

we put $y=z=u$ and obtain $(u v)^{2}=0$ for all $v \in A$. Hence $u$ belongs to the radical of $A$ and therefore has to be zero. It follows immediately that $K[x]$ does not contain any nonzero nilpotent elements and as a finite field extension of $K e$ coincides with $K e$. Theorem 1 is thereby proved.

COROLlaRY. Let $x$ be a nonzero-divisor in the semisimple Jordan algebra $A$ over $K$ such that $L^{-1}(x)=L\left(x^{-1}\right)$. Then $x$ belongs to the center of $A$. 
We are following the invertibility concept of [2].

Proof. According to $[2$, p. 90$]$, we have $L(x)=P(x) \circ L\left(x^{-1}\right)$ $=P(x) \circ L^{-1}(x)$ or $P(x)=L^{2}(x)$. Now Theorem 1 shows $x \in Z(A)$.

2. We shall now present a more detailed version of Theorem 2 , viz.

Theorem $2^{\prime}$. Let $A, B$ be Jordan algebras over $K, A$ be semisimple and $\rho, \sigma, \tau$ be bijective linear mappings from $B$ onto $A$ such that $\rho(x) \sigma(y)$ $=\tau(x y)$ for all $x, y \in B$. Then there are invertible elements $u, v$ in the center of $A$ and an isomorphism $\phi$ from $B$ onto $A$ such that $\rho=L(u) \circ \phi$, $\sigma=L(v) \circ \phi, \tau=L(u v) \circ \phi$.

We split the proof into several steps.

(a) First we show that $B$ is semisimple; for otherwise it is well known that $B$ contains a nonzero ideal $\mathfrak{b}$ with $\mathfrak{b}^{2}=0$. Since $A$ contains an identity element $e$, it is clear according to [1, p. 699], that we have $\rho(\mathfrak{b})=\sigma(\mathfrak{b})=\tau(\mathfrak{b})$ and this is a nonzero ideal in $A$. It follows $\sigma(\mathfrak{b})^{2}=\rho(\mathfrak{b}) \sigma(\mathfrak{b})=\tau\left(\mathfrak{b}^{2}\right)=0$, a contradiction to the semisimplicity of $A$.

(b) In particular, $B$ contains an identity element $f$ and after what we have just said it is obvious that the triple $(\rho, \sigma, \tau)$ maps the ideals of $B$ in a one-to-one fashion onto the ideals of $A$. Hence, considering the simple components of both algebras justifies the assumption that $A$ and $B$ are simple. Clearly, the elements $u=\rho(f), v=\sigma(f)$ are nonzero-divisors in $A$, and it suffices to show that they belong to the center. Indeed, this would imply the properties $\rho^{\prime}(x) \sigma^{\prime}(y)=\tau^{\prime}(x y)$ $(x, y \in B)$ and $\rho^{\prime}(f)=\sigma^{\prime}(f)=\tau^{\prime}(f)=e$, for the mappings $\rho^{\prime}=L(u)^{-1} \circ \rho$, $\sigma^{\prime}=L(v)^{-1} \circ \sigma, \quad \tau^{\prime}=L(u v)^{-1} \circ \tau$; consequently, we would obtain $\rho^{\prime}=\sigma^{\prime}=\tau^{\prime}$ and this would be an isomorphism $\phi$ from $B$ onto $A$ satisfying the desired conditions.

(c) We now study the map $\omega=\rho \circ \sigma^{-1}$ from $A$ onto itself. We have $\rho(x) \sigma(y)=\rho(y) \sigma(x)$ for all $x, y \in B$ and obtain

$$
\rho(x)=\rho(x) \sigma\left(\sigma^{-1}(e)\right)=\omega(e) \sigma(x) .
$$

From

$$
(\sigma(x) \omega(e)) \sigma(y)=\rho(x) \sigma(y)=\rho(y) \sigma(x)=(\sigma(y) \omega(e)) \sigma(x)
$$

for all $x, y \in B$ we conclude that $\omega(e)$ belongs to the middle nucleus of $A$. Since $A$ is semisimple, (3) for $\sigma^{-1}(x)$ instead of $x$ gives $\omega=L(w)$ where $w$ denotes an invertible element in $Z(A)$.

(d) By symmetry it suffices now to establish $v$ as an element in the center. Evidently we have $\tau=L(v) \circ \rho=L(u) \circ \sigma$ which implies $\omega=L(v)^{-1} \circ L(u)$. Hence we get 


$$
\begin{aligned}
\omega(\sigma(x y)) & =L(v)^{-1}(\tau(x y))=L(v)^{-1}(\rho(x) \sigma(y))=L(v)^{-1}(L(w)(\sigma(x) \sigma(y))) \\
& =\omega\left(L(v)^{-1}(\sigma(x) \sigma(y))\right)
\end{aligned}
$$

for all $x, y$ in $B$. Putting $\psi=L(v)^{-1}$ we see that $\sigma(x y)=\psi(\sigma(x) \sigma(y))$. Making use of this and the identity $\sigma\left(x\left(x^{2} y\right)\right)=\sigma\left(x^{2}(x y)\right)$ one easily derives the functional equation

$$
\psi\left(x^{2}\right) \psi(x y)=x \psi\left(\psi\left(x^{2}\right) y\right)
$$

for $\psi$ identically in $x, y \in A$. Linearization yields

$$
2 \psi(x z) \psi(x y)+\psi\left(x^{2}\right) \psi(z y)=z \psi\left(\psi\left(x^{2}\right) y\right)+2 x \psi(\psi(x z) y)
$$

for all $x, y, z \in A$. Now let $c$ be an idempotent in $A$; we write $A_{\lambda}$ $=A_{\lambda}(c)$ for $\lambda=0, \frac{1}{2}, 1$ and consider elements $x \in A_{1}, y \in A_{0}$. (4') for $x=c$ and $z=x$ yields $x \psi(\psi(c) y)+2 c \psi(\psi(x) y)=0$. On the other hand, (4) with $x=c$ proves $\psi(\psi(c) y)$ to belong to $A_{0}$ and hence we obtain

$$
\psi(\psi(x) y) \in A_{0} \quad\left(x \in A_{1}, y \in A_{0}\right) .
$$

Considering $e-c$ instead of $c$, we also have

$$
\psi(\psi(x) y) \in A_{1} \quad\left(x \in A_{0}, y \in A_{1}\right) .
$$

Now we take elements $x \in A_{0}, y \in A_{1}$, put $z=c$ in $\left(4^{\prime}\right)$ and observe $\left(5^{\prime}\right)$. After linearizing the result is $\psi\left(x_{1} x_{2}\right) \psi(y)=\psi\left(\psi\left(x_{1} x_{2}\right) y\right)$ for $x_{1}, x_{2}$ in $A_{0}$, hence in particular

$$
\psi(\psi(x) y)=\psi(x) \psi(y) \quad\left(x \in A_{0}, y \in A_{1}\right) .
$$

Similarly,

$$
\psi(\psi(x) y)=\psi(x) \psi(y) \quad\left(x \in A_{1}, y \in A_{0}\right) .
$$

The identities (5)-(6') now yield for $x \in A_{1}, y \in A_{0}: \psi(x) \psi(y)=\psi(\psi(x) y)$ $=\psi(\psi(y) x) \in A_{0} \cap A_{1}$ or $\psi(x) y=0$. Hence $\psi$ and therefore $L(v)$ maps the subspace $A_{1}$ onto itself. Summing up, we obtain that $v$ belongs to $A_{1}(c)+A_{0}(c)$ for each idempotent $c$ in $A$.

(e) In order to show $v \in Z(A)$, we may assume that $A$ is central simple over $K$, since $\psi$ commutes with the maps $L(z), z \in Z(A)$. Moreover, (5) remains valid in every base field extension of $A$, which justifies the additional assumption of $K$ being algebraically closed. Then we choose a complete orthogonal system $\left\{c_{1}, \cdots, c_{r}\right\}$ of primitive idempotents in $A$ which induces a Peirce decomposition $A=\sum_{i \leq j} A_{i j}$ of $A$ with the well-known multiplication properties of the subspaces $A_{i j}\left[2\right.$, p. 241]. According to (d) we have $v=\sum_{k=1}^{r} \alpha_{k} c_{k}$ with scalars $\alpha_{1}, \cdots, \alpha_{r}$, since $A_{1}\left(c_{k}\right)=A_{k k}=K c_{k}[2, \mathrm{p} .156]$. As is well known, 
there is an element $a_{i j} \in A_{i j}$ such that $a_{i j}^{2}=c_{i}+c_{j}$ for a fixed pair of indices $i, j$. If we put $e_{i}=\frac{1}{2}\left(c_{i}-a_{i j}+c_{j}\right), e_{j}=\frac{1}{2}\left(c_{i}+a_{i j}+c_{j}\right)$ and $e_{k}=c_{k}$ for $k \neq i, j$, we obtain a complete orthogonal system $\left\{e_{1}, \cdots, e_{r}\right\}$ of idempotents which are all primitive $[2$, p. 119]. Hence we get $v=\sum_{k=1}^{r} \beta_{k} e_{k}$ with certain scalars $\beta_{1}, \cdots, \beta_{r}$. Comparing these two equations for $v$ one obtains $\alpha_{i}=\frac{1}{2}\left(\beta_{i}+\beta_{j}\right)=\alpha_{j}$ and therefore $v=\alpha e$ for some $\alpha \in K$. This proves the theorem.

3. Let $A$ be an arbitrary algebra with identity element $e$ over $K$. We shall call a bijective linear map $\eta$ from $A$ onto itself a semi-automorphism of $A$ providing $\eta(x) \eta(y)=\eta(e) \eta(x y)$ for all $x, y \in A$. One verifies immediately that the set $H(A)$ of all semi-automorphisms of $A$ forms a subgroup of $\mathrm{GL}(A)$ which acts on the left nonzero-divisors of $A$ in the natural fashion. Now let $A$ be a semisimple Jordan algebra over $K$ and $\eta \in H(A)$. Then the map $\psi=L(v)^{-1}$ with $v=\eta(e)$ is well defined, and if $B$ denotes the algebra that is given by the multiplication $x \cdot y=\psi(x y)$ on the underlying vector space of $A$, we obtain $\eta(x) \cdot \eta(y)=\psi(\eta(x) \eta(y))=\psi(\eta(e) \eta(x y))=\eta(x y)$ for all $x, y$ in $A$. Hence $\eta$ is an isomorphism from $B$ onto $A$, and $B$ is a Jordan algebra. In particular, $\psi$ satisfies the functional equation (4). Therefore, steps (d) and (e) in the preceding proof give $v=\eta(e) \in Z(A)$. Summing up, we obtain that every semi-automorphism of $A$ can be written in the form $L(v) \circ \phi$ with an invertible element $v$ in the center and an automorphism $\phi$ of $A$.

Finally, let $A$ be a commutative algebra with identity element $e$ over $K$. We are referring to the invertibility concept introduced by $\mathrm{M}$. Koecher in [3], choose a generic element $x$ of $A$ and denote by $\Gamma(A)$ the structure group of $A$, i.e., the set of those elements $\phi \in \mathrm{GL}(A)$ such that there is a $\phi^{\#} \in \mathrm{GL}(A)$ satisfying $\phi(x)^{-1}=\left(\phi^{\#}\right)^{-1}\left(x^{-1}\right)$. According to [3], the map $\Gamma(A) \rightarrow \Gamma(A), \phi \mapsto \phi^{\sharp}$, is well defined and an involution of $\Gamma(A)$. In particular, the set $\Gamma_{1}(A)$ of elements $\phi \in \Gamma(A)$ for which $\phi^{*}=\phi^{-1}$, is a subgroup of $\Gamma(A)$. The following statement describes the relationship of the groups $H(A)$ and $\Gamma_{1}(A)$.

Proposition. A bijective linear map $\eta$ from $A$ into itself belongs to $\Gamma_{1}(A)$ if and only if $\eta$ is a semi-automorphism of $A$ such that $\eta(e)^{2}=e$.

Proof. First, let $\eta \in H(A)$ and $\eta(e)^{2}=e$. Then we have $\eta(x) \eta(y)$ $=\eta(e) \eta(x y)$ for generically independent elements $x, y$ over $K$, and the specialization $y \mapsto x^{-1}$ yields $\eta \in \Gamma_{1}(A)$. Conversely, let this be the case and $T$ be an indeterminate over $K$. If $A^{\prime}$ denotes the algebra that we obtain by extending the base field $K$ to $K(T)$, then $e+T u$ 
evidently is invertible in $A^{\prime}$ for each $u \in A$, and the inverse admits the expansion

$$
(e+T u)^{-1}=e-T u+T^{2} u^{2}+O\left(T^{3}\right),
$$

where $O\left(T^{3}\right)$ stands for a rational function in $T$ with numerator (in reduced representation) divisible by $T^{3}$ and denominator prime to $T$. We have $e=\eta(e+T u) \eta\left((e+T u)^{-1}\right)$, and using the above expansion we conclude $\eta(e) \eta\left(u^{2}\right)=\eta(u)^{2}$ or $\eta \in H(A)$.- If $A$ is a simple Jordan algebra, we may combine this proposition with the previous characterization of $H(A)$ and obtain the fact, known from [2], that $\Gamma_{1}(A)$ consists of the elements $\pm \phi$ where $\phi$ is running over the automorphism group of $A$.

\section{REFERENCES}

1. A. A. Albert, Nonassociative algebras. I, Ann. of Math. 43 (1942), 685-707.

2. H. Braun and M. Koecher, Jordan-Algebren, Springer, New York, 1966.

3. M. Koecher, On homogeneous algebras, Bull. Amer. Math. Soc. 72 (1966), 347357.

4. R. H. Oehmke and R. Sandler, The collineation groups of division ring planes. I. Jordan algebras, J. Reine Angew. Math. 216 (1964), 67-87.

The UNIVERsity OF IOWA 